\title{
La "industria" de la evaluación y bibliografía básica
}

\section{Xavier Ballart*}

\section{La «ndustria" de la evaluación}

Se puede hablar con propiedad de una verdadera industria de la evaluación para referirse al sector tanto académico como profesional que se ha desarrollado durante los últimos veinte años al calor de la demanda de estudios de organizaciones internacionales -Banco Mundial, agencias de Naciones Unidas, Unión Europea - gobiernos nacionales - principalmente Estados Unidos y, de forma creciente, los gobiernos europeosadministraciones regionales y locales. El sector cuenta con dos grandes organizaciones profesionales, una en Estados Unidos y otra en Europa, donde se reúnen académicos de procedencias muy diversas -psicometría, econometría, sociología, educación, analistas de políticas públicas- además de profesionales de la auditoría y representantes de las agencias y tribunales de cuentas. La European Evaluation Society, creada recientemente, tiene un marcado caractér profesional frente a la sociedad norteamericana, donde tienen una mayor presencia los académicos.

Existe un número considerable de publicaciones epecializadas en evaluación. De hecho, hay revistas especializadas en evaluación en un campo sectorial como, por ejemplo, Educational Evaluation and Policy Analysis. Las tres publicaciones que representan mejor al campo son, sin embargo:

1. Evaluation Jourmal, norteamericana, con una orientación muy cuantitativa y académica.

2. Evaluation and Program Planning, con una orientación más internacional, intenta combinar aproximaciones cuantitativas y cualitativas.

3. Evaluation, The International Journal of Theory, Research and Practice, la revista europea con orientación interna- cional, que intenta combinar aproximaciones académicas y profesionales.

\section{Principales referencias bibliográficas en evaluación ${ }^{1}$}

La evaluación de políticas es a la vez una "ciencia" y un "arte" dadas las limitaciones que encuentra una aproximación puramente científico-técnica positivista en un contexto real. Ello no significa que la evaluación de programas carezca de una base teórica o que cualquier forma de análisis pueda ser aceptada como una forma válida de evaluación ${ }^{2}$. Ante la diversidad de los estudios en evaluación, cabe distinguir entre una abundante literatura sobre métodos y una literatura sobre utilización de las evaluaciones y sobre inserción de sus resultados en el proceso político-administrativo. Sin embargo, también puede incluirse dentro del campo de la evaluación, dada su relevancia a nivel práctico, la literatura de control gestión y que relaciona el campo de la evaluación con el análisis de procesos, calidad y reingeniería, y con los sistemas de información contable-financiera o de control de operaciones, propios de la gestión pública o privada y del análisis de organizaciones.

\section{Evaluación como técnica de investigación social aplicada}

Una buena parte de la literatura sobre evaluación tiene por objeto obtener un mejor conocimiento tanto de los procesos de 
aplicación como de los resultados de la intervención pública en la sociedad. Se reproduce aquí el debate entre aproximaciones cuantitativas y cualitativas propio de la investigación aplicada en ciencias sociales.

Por una parte, existe una amplia literatura sobre análisis de causalidad, validez interna y externa, fiabilidad, series temporales, grupos de comparación, encuestas y muestreo, análisis por métodos estadísticos..., que se presenta en obras monográficas (COOK y CAMPBELL, 1979, sobre cuasi-experimentos; CARMINES y ZelLER, 1979, sobre validez y fiabilidad; Fowler, 1993 sobre encuestas; Kanjl, 1993, sobre tests estadísticos) o en obras generales (Rossi y Freeman, 1989; MoHr, 1988; Light, Singer, y WILLETT, 1990; PosaVac y CaReY, 1992; WeISS, 1997).

Por otra parte, existe una literatura que se fundamenta en métodos cualitativos y que tiene su origen en las obras de ScRIVEN sobre los valores en evaluación (SCRIVEN, 1980), STAKE y GuBA y LinColn (Stake 1978; GuBa y LinColn, 1989), sobre evaluación natural y responsive, también llamada de "cuarta generación". Otras obras cualitativas más generales son International Innovations in Evaluation Methodology: New Directions for Program Evaluation, editado por CONNER y HENDRICKS (1989), o el mismo Handbook of Qualitative Research, editado por DENZIN y LINCOLN (1994). Algunos autores significativos de ambas corrientes han propuesto la integración de los métodos, como puede verse en The Qualitative-Quantitative Debate: New Perspectives, editado por REICHARDT y RALLIS en 1994, en especial los trabajos de House, Rossi y SMITH.

\section{Evaluación como instrumento de negociación en el proceso político}

Una parte emergente de la literatura sobre evaluación se centra en los problemas de utilización de los resultados. Ello se suele relacionar con los métodos en la medida en que éstos afectan de forma directa la interacción entre comanditarios de las evaluaciones, analistas y sujetos evaluados. Esta orientación parte de los trabajos de Carol WeIss sobre el entorno políticoadministrativo de la evaluación y del libro editado por BRYK en 1983, Stakebolder-Based Evaluation, New Directions for Program Evaluation, en especial, la contribución de WeISS a este libro. Sobre política y evaluación, la obra fundamental es la de PALumBO (1987), mientras que sobre utilización de las evaluaciones, en 1989 MCLAughuIN, WeBER, CoverT e INGLE editaron un nuevo volumen de New Directions for Program Evaluation sobre esta cuestión. Con anterioridad, PATTON (1989) había publicado ya varias ediciones de un libro que dio origen a una nueva modalidad de evaluación -Utilization-Focussed Evaluation - que se caracteriza por poner menos énfasis en la excelencia de los métodos y más en la discusión de las formas de organizar el proceso evaluador. Esta nueva forma de plantear la evaluación, hasta el punto de considerarla como un elemento de negociación antes que como una forma de análisis de los resultados de una política, tuvo un considerable éxito tanto en Estados Unidos como en Europa. En castellano, puede verse el libro de MONNIER (1995).

\section{Evaluación e indicadores: control de gestión}

La práctica de la evaluación por las Administraciones públicas tiene, sin embargo, mayores puntos de contacto con los indicadores de costes, operaciones, actividades y resultados de los sistemas de control de gestión, que con los análisis, ya sean cuantitativos o cualitativos, propios de la investigación social aplicada.

La literatura sobre contabilidad de costes es muy amplia y puede encontrarse en la bibliografía específica de esta disciplina. Sin embargo, algunas publicaciones recogen la adaptación de nuevas formas de contabilidad a las características específicas del sector público y a los programas de innovación en la gestión. Por ejemplo, en el caso del programa de ureinvención del gobiernon estadounidense, KeHOE, Dodson, ReEve y Plato desarrollaron Activity-Based Management in Government, 1995 como una nueva forma de control de gestión.

Si bien los recursos que utiliza una organización siempre pueden traducirse a unidades monetarias y, por tanto, a costes, resulta mucho más dificil medir outputs e impactos. En el sector privado, los ingresos por la venta de los productos son, en sî mismos, una buena medida de output, aunque tampoco incluyen actividades que pueden tener un valor social, como, por ejemplo, la disminución en la contaminación del medio ambiente. En el sector público, resulta casi imposible tener una buena medida de resultado final. Así, por ejemplo, si bien es posible cuantificar el número de estudiantes que finalizan el curso un determinado año, resulta más difícil determinar cuánta educación obtuvo cada uno de ellos. Además, una misma organización pública suele prestar varios servicios de características muy distintas, con lo que resumir los distintos "productos" en una sola medida final, como suelen hacer las organizaciones privadas, es casi imposible. En el mismo sentido, la idea de calidad se refiere a algo que escapa a la cuantificación de los out puts o de los ingresos y que a menudo depende estrictamente del juicio individual de las personas. Otros efectos intangibles como la imagen de una organización, el sentido de lealtad o el 
orgullo que pueda generar, por ejemplo, un programa de formación, son muy difíciles de medir. Sobre las dificultades de medir rendimiento en el sector público: WhOLEY, ABRAMSON y

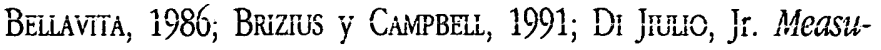
ring Performance When There is No Bottom Line, 1994.

De ahí la importancia de la evaluación de programas como conjunto de técnicas que intentan refinar las medidas y obtener información, ya sea sobre el producto abruto de las políticas, o, en otros casos, sobre su impacto neto en sus beneficiarios directos o en la sociedad. Ésta es una literatura propia del sector público, cuyo equivalente en el sector privado suele tener metas menos ambiciosas y se limita estrictamente a la contabilidad de costes y a la medida de actividades y productos finales como puede verse en ANTHONY y Young, Management Control in Nonprofit Organizations, 1988; Young, 1984 y HONGREN y FosTER, Cost Accounting: A Managerial Emphasis, 1987. Si bien la literatura de control de gestión para el sector público específicamente o para el tercer sector es bastante minoritaria -la principal obra es la citada de ANTHONY y Young - hay algunos trabajos en castellano que merecen una referencia. Por ejemplo, para la administración local, LOPEZ y GADEA (1992) o en general para las administraciones públicas, el cuaderno presentado recientemente por el colectivo de auditores contables (AECA, 1997) o las experiencias y casos recogidos en los números 3 y 5-6 de esta misma revista (GIró y RoCA, 1995; ZaPICO y MAYNE, 1995; Fuentes Vega, 1996; Del Barco Fernández Molina, 1996; Ballart y Ramió, 1996).

\section{Evaluación, obras generales y métodos cuantitativos}

Alvira MarTín, F. (1991), Metodología de la evaluación de programas. Centro de Investigaciones Sociológicas, Madrid.

Ballart, X. (1992), ¿Cómo Evaluar Programas y Servicios Públicos? Aproximación Sistemática y Estudios de Caso. Madrid, Ministerio para las Administraciones Públicas, 1992.

- (1995), "Impact of legislation requiring moped and motorbike riders to wear helmetsn, Evaluation and Program Planning; vol. 18, n. ${ }^{4}$. En castellano, en Hacienda Pública Española, 135.

- (1996), «Modelos teóricos para la práctica de la evaluación de programas", Lecturas de Gestión Pública, INAPBOE, Madrid.

- (1998), "Spanish Evaluation Practice vs. Program Evaluation Theory", Evaluation. The International Journal of Theory, Research and Practice, vol. 4, n. 2 .
CARMines, E.G. y ZelleR, R.A. (1979), Reliability and Validity Assessment, Newbury Park, Ca: Sage.

COOK, T.D. y CAMPBELL, D.T. (1979), Quasi-experimentation: Design and Anaiysis issues for Field Selitings. Boston, Mã: Houghton Mifflin.

Fowler, F.J. (1993), Survey Research Methods ( $2^{\text {nd }}$ edition). Newbury Park, Ca: Sage.

KanjI, G. (1993), 100 Statistical Tests. Thousand Oaks, Ca: Sage.

Light, R.T.; Singer, J.D. y Willett, J.B. (1990), By Design. Cambridge, Ma: Harvard University Press.

MoHR, L.B. (1988), Impact Analysis for Program Evaluation. Chicago, Ill.:The Dorsey Press.

POSAVAC, E.I. y CaREY, R.G. (1992), Program Evaluation, Metbods and Cuse Studies (fourth edition). Englewood Cliffs. N.J.: Prentice Hall.

Rossi, P.H. y Freeman, H.E. (1989), Evaluación. Un enfoque sistemático para programas sociales. México: Trillas, 1990.

SHADISH, W.R. y EPSTEIN, R. (1987), "Patterns of Program Evaluation Practice. Among Members of the Evaluation Research Society and Evaluation Network. Evaluation Review, 11, p. 555.

- ; Cook, T.D. y Leviton. L.C. (1991), Foundations of Program Evaluation: Theories of Practice. Newbury Park, Ca: Sage.

WeIss, C.H. (1972), Evaluation Research. Upper Saddle River, NJ.: Prentice Hall.

\section{Evaluación, métodos cualitativos}

CONNER, R.F. y HENDRICKS, M. (1989), International Innovations in Evaluation Methodology: New Directions for Program Evaluation.

DENZIN, N.K. y Lincoln, Y.S. (1994), Handbook of Qualitative Research. Newbury Park, Ca: Sage.

GubA, E. y Lincoln, Y.S. (1989), Fourth Generation Evaluation. Newbury Park, Ca: Sage.

REICHARDT, C. y RALLIS, S. (1994), The Qualitative-Quantitative Debate: New Perspectives. New Directions for Program Evaluation. N.. 61 .

SCRIVEN, M. (1980), The Logic of Evaluation. Inverness, Ca: Edgepress.

STAKE, R.E. (1978), "The Case Study Method in Social Inquiry", Educational Researcher, 7. 


\section{Evaluación y proceso politico}

BRYK, A. (1983), Stakebolder-Based Evaluation. New Directions for Program Evaluation. N. 17.

MClaughin, J.; Weber, l.; CoverT, R. y Ingle, R. (1989), Evaluation Utilization. New Directions for Program Evaluation. N. 39.

MONNIER (1995), Evaluación de la Acción de los Poderes Públicos. Madrid: Instituto de Estudios Fiscales.

Palumbo, D. (1987), The Politics of Program Evaluation. Newbury Park, Ca: Sage.

PatTon, M. (1989), Utilization-Focussed Evaluation (second edition). Newbury Park, Ca: Sage.

WEISS, C. (1983), "The Stakeholder Approach to Evaluation: Origins and Promise" $y$ "Toward the Future of Stakeholder Approaches in Evaluation". En A. BRYK, Stakebolder-Based Evaluation. New Directions for Program Evaluation, ... 17.

\section{Control de gestión}

AECA (1997), "Indicadores de Gestión para las administraciones públicas". Contabilidad de Gestión, n.․ำ 16.

ANTHony, R. y Young, D. (1988), Management Control in Nonprofit Organizations. I.L. HOMEWOOD, Dow-Jones-Irwin, cuarta edición.

Ballart, X. y Ramió, C. (1996), "Indicadores para el control de gestión de personal. Gestión y Análisis de Políticas Públicas, n. ${ }^{\text {os }} 5-6$.
Brzzius, J.A. y CAMPBELL, M.D. (1991), Getting Results: A Guide for Government Accountability. Washington DC: Council of Governors' Policy Advisors.

Del Barco Fernández Molina (1996), "La estrucutra de control en el contexto del sistema normalizado de seguimiento de objetivos". Gestión y Análisis de Politicas Públicas, n. ${ }^{\text {ss }}$ 5-6.

Dilulio, J.Jr. (1994), Measuring Performance When There is No Bottom Line. Center for Public Management Woring Paper 94-2, Washington DC.: The Brookings Institutiton.

FuENTES VeGA, S. (1996), "La contabilidad analítica y la medida de la eficiencia en los servicios públicos", 1996. Gestión y Análisis de Politicas Públicas, n. ${ }^{\text {os }}$ 5-6.

GIRÓ RoCA, J.M. (1995), “El control de gestión en el antiguo MOPT. Los límites de una visión gerenciall. Gestión y Análisis

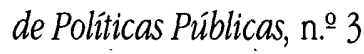

Hongren, C. y Foster, G. (1987), Cost Accounting: A Managerial Emphasis. Englewood Cliffs, NJ. Prentice Hall.

Kehoe, J.; Dodson, W.; Reeve, R. y Plato, G. (1995), ActivityBased Management in Government. Washington DC: Coopers and Lybrand.

LOOPEZ, J. y GADEA, A. (1992), El Control de gestión en la Administración Local. Gestión 2000, Barcelona.

Young, D.W. (1984), Financial Control in Health Care: A Managerial Perspective. I.L. HOMEwOOD, Dow Jones-Irwin.

Wholey, J.S.; ABRamson, M.A. y Bellavita, C. (1986), Performance and Credibility: Developing Excellence in Public and Nonprofit Organizations. Lexington, Ma: DC Heath.

ZAPICO GoÑ, E. y MAYNE, J. (1995), "Nuevas perspectivas para el control de gestión y medición de resultados". Gestión y Análisis de Políticas Públicas, n. 3 .

\footnotetext{
- Universidad Autónoma de Barcelona.

${ }^{1}$ Este comentario reproduce, en parte, el articulo bibliográfico del mismo autor publicado en la Revista de Estudios Políticos, n..97, 1997, bajo el título :Gestión Pública, Análisis y Evaluación de Políticas. Delimitación de contenidos y bibliografia.

2 Para una delimitación teórica del campo, la obra principal es SHADISH, COOK y LEVITON (1991). En castellano, puede verse mi artículo sobre modelos teóricos
}

donde además de explicar las aportaciones de siete autores fundamentales, se analizan estas aportaciones en función de nueve categorías conceptuales (BALLART, 1996). También cabe recordar que SHADISH y EPSTEIN habían realizado en 1987 un interesante análisis -composición sociológica, por disciplinas, orientaciones teóricas y prácticas- de los asistentes a los principales congresos internacionales de evaluación de políticas. 